\title{
Percentage of motile spermatozoa at 22 hours after swim-up procedure: An indicator for intracytoplasmic sperm injection?
}

\author{
Taketo Inoue ${ }^{1,2}$, Yukiko Yonezawa ${ }^{1}$, Hironobu Sugimoto ${ }^{1}$, Mikiko Uemura $^{2}$, Yuri Ono' , Junji Kishi ${ }^{1}$, Nobuyuki Emi ${ }^{1}$, Yoshiyuki Ono \\ 'Ono Ladies Clinic, Ono; ${ }^{2}$ Department of Biophysics, Kobe University Graduate School of Health Sciences, Kobe, Japan
}

Objective: The decision to use in vitro fertilization (IVF), intracytoplasmic sperm injection (ICSI), or split insemination (IVF-ICSI) in the first cycle is based on the number of motile sperm. Hence, total fertilization failure (TFF) often occurs during IVF cycles, despite normozoospermia. To investigate whether the cumulative motile swim-up spermatozoa percentage at 22 hours post-insemination (MSPPI) is an indicator for ICSI, we analyzed TFF, fertilization, blastocyst development, chemical pregnancy, clinical pregnancy, and live birth rates.

Methods: This prospective study was performed using data obtained from $260 \mathrm{IVF}$ cycles. At 22 hours after insemination, the remaining swimup spermatozoa were observed and divided into six groups according to MSPPI ( $<10 \%, 10 \%$ to $<30 \%, 30 \%$ to $<50 \%, 50 \%$ to $<70 \%, 70 \%$ to $<90 \%$, and $90 \%$ to $100 \%)$.

Results: Regardless of the ejaculated motile sperm concentration $\left(0.6-280 \times 10^{6} / \mathrm{mL}\right.$ motile spermatozoa), the incidence of TFF significantly increased when MSPPI was $<10 \%$, and the fertilization rate significantly decreased when MSPPI was $<30 \%$. We found that cumulative MSPPI correlated with the cumulative fertilization rate (Spearman correlation, $0.508, p<0.001$ ). Regarding embryo development, we observed no significant differences in the rates of blastocyst development, chemical pregnancy, clinical pregnancy, or live birth among all groups.

Conclusion: Our findings suggest that MSPPI is a viable indicator for split IVF-ICSI and ICSI. Taken together, by employing the MSPPI test in advance before IVF, ICSI, or split IVF-ICSI cycles, unnecessary split IVF-ICSI and ICSI may be avoided.

Keywords: Fertilization in vitro; Insemination; Sperm injections, intracytoplasmic; Spermatozoa

\section{Introduction}

Total fertilization failure (TFF) occurs in 5\%-25\% of conventional in vitro fertilization (IVF) cycles [1-3]. The main causes of TFF and low fertilization rate, defined as a fertilization rate of $<25 \%$ during IVF, are defective sperm-zona pellucida binding or sperm penetration, which are mainly due to abnormalities in the sperm and not in the oocytes themselves [4].

To avoid a complete failure of IVF, rescue intracytoplasmic sperm injection (ICSI) [5-8] and split insemination (IVF-ICSI) [9] are often

Received: May 5, 2016 · Revised: Jul 26, 2016 · Accepted: Aug 8, 2016 Corresponding author: Taketo Inoue

Ono Ladies Clinic, 538-3, Nishihonmachi, Ono, Hyogo 675-1375, Japan

Tel: +81-794-621103 Fax:+81-794-621106 E-mail: taketoein@yahoo.co.jp

This is an Open Access article distributed under the terms of the Creative Commons Attribution Non-Commercial License (http://creativecommons.org/licenses/by-nc/4.0/) which permits unrestricted non-commercial use, distribution, and reproduction in any medium, provided the original work is properly cited. performed. The decision to use IVF, ICSI, or split IVF-ICSI is based on the number of motile sperm evident in the first cycle. A European Society of Human Reproduction and Embryology position paper [10] suggested that ICSI should be considered in cases of severe sperm abnormalities or in patients with a history of fertilization failure with conventional IVF attempts. However, ICSI is not the most suitable treatment for pathologies in women, such as poor ovarian response or previous implantation failures. This is consistent with the opinions of the Practice Committees of the American Society for Reproductive Medicine and Society for Assisted Reproductive Technology [11], and the Japan Society of Obstetrics and Gynecology [12]. Therefore, unnecessary ICSI should be avoided. However, because these committees do not provide concrete criteria (e.g., numbers of motile sperm) regarding the indications for ICSI, each hospital/clinic follows its own ICSI criteria. Our ICSI criteria comprise the following: (1) after sperm preparation, when no progressive motile spermatozoa are observed (e.g., sper- 
matozoa in which only a flagellar beat can be observed); (2) after sperm preparation, when insufficient numbers of progressive motile spermatozoa are obtained ( $<20,000$ spermatozoa/oocyte); (3) IVF has failed completely twice in a row (in case of TFF, at first cycle, IVF is performed, at second cycle, split IVF-ICSI is performed, and at third cycle, ICSI is performed); or (4) patients strongly request ICSI.

Rescue ICSI complies with the need to avoid unnecessary ICSI. Previous studies have reported that rescue ICSI is performed at 22 hours [5], 6 hours [7], or 3 hours [8] post-insemination. The fertilization and implantation rates of rescue ICSI were significantly lower after 22 hours than after 6 hours because of oocyte aging [7]. A cost-effectiveness analysis of rescue ICSI showed a $25 \%$ reduction in the cost per live birth, even when rescue ICSI was performed at 1 day after insemination, compared with cycle cancelation [13]. Accordingly, early rescue ICSI, performed as early as 6 hours after insemination, is an effective backup treatment to avoid TFF [6]. However, the rate of polyspermy was significantly higher with early rescue ICSI than with ICSI [14]. Moreover, Vitek et al. [15] demonstrated that IVF is preferable because of its increased live birth rate relative to split IVF-ICSI and ICSI in a single cycle. Therefore, to avoid unnecessary ICSI, a predictor of fertilization is crucial.

Coccia et al. [16] reported that a sperm survival test (SST) may predict the outcome of IVF in the presence of male factor infertility. In contrast, several studies have asserted that the percentage of sperm motility at 24 hours and the sperm survival ratio in both semen and sperm suspension for insemination have no practical value in predicting fertilization in vitro $[17,18]$. Eskandar [18] reported that the fertilization rate decreases when the percentage of motile spermatozoa is $0 \%$ at 24 hours after insemination. In our study, we focused on the relationship between the motile swim-up spermatozoa percentage at 22 hours post-insemination (MSPPI, our calculation method differed from that of the SST) and on the fertilization rate, because we have rarely observed motile sperm during 1 day-old rescue ICSI at 22 hours after insemination in patients with TFF and low fertilization rate (unpublished data). The relationship between SST and the fertilization rate has been investigated, but few studies have discussed single sperm analysis and the impact on embryo development/pregnancy rates. In this study, we investigated the relationship between the cumulative MSPPI and fertilization/embryo development/pregnancy rates.

\section{Methods}

\section{Patients}

This prospective study was performed using data obtained from 260 IVF cycles performed between July 2013 and July 2014 and frozen blastocyst transfer (FBT) cycles derived from these IVF cycles. In- formed consent was obtained for all procedures. All procedures were performed in accordance with the Declaration of Helsinki.

\section{Ovarian stimulation}

Ovarian stimulation was performed as previously described [19]. Briefly, long, short, or gonadotropin-releasing hormone (GnRH)-antagonist protocols were used depending on the patient. In poor responders who have $\leq 2$ follicles even if they were injected with human menopausal gonadotropin (hMG; ASKA Pharmaceutical Co., Tokyo, Japan) several times before during previous infertility treatment, including timing treatment or artificial insemination with sperm from the patient's husband, in combination with herbal medicine, clomifene citrate (50 mg Spacromin tablet, Pola Pharma Inc., Tokyo, Japan) and hMG were used for ovarian stimulation [20]. A GnRH analogue acetate (Fuji Pharma Co., Tokyo, Japan), hMG, and a GnRH antagonist (Cetrotide, Shionogi \& Co., Osaka, Japan) were used in each protocol. When at least two follicles reached 18 to $20 \mathrm{~mm}$ in diameter (as determined by transvaginal ultrasonography), 5,000 IU of human chorionic gonadotropin (Fuji Pharma Co.) was administered. Ovum pickup was performed 35 hours after the human chorionic gonadotropin injection.

\section{Collection of sperm/IVF}

Ejaculated sperm were collected and incubated for at least 1 hour. Semen samples were analyzed using a Makler Counting Chamber (Sefi Medical Instruments, Haifa, Israel). Semen samples were overlaid on to $90 \%$ ISolate (Irvine Scientific, Santa Ana, CA, USA) and then centrifuged at 1,000 revolutions per minute (rpm) for 10 minutes (luchi Centrifuge CN-10, AS ONE, Osaka, Japan). The upper layers were aspirated, and $1 \mathrm{~mL}$ of Universal IVF Medium (Origio, Måløv, Denmark) was added and pipetted with the pellet. The sperm sample was centrifuged at 1,000 rpm for 5 minutes. The supernatant was removed, and 0.5 to $1 \mathrm{~mL}$ of Universal IVF Medium was added to the pellet. Sperm samples were subjected to swim-up procedure for 10 minutes in an incubator. Swim-up spermatozoa were obtained. Approximately $10 \times 10^{4} / \mathrm{mL}$ swim-up sperm were added to a one-well IVF dish (Falcon, Corning Life Sciences, Tewksbury, MA, USA) containing $<5$ cumulus oocyte complexes in $1 \mathrm{~mL}$ of Universal IVF Medium. If no progressive motile spermatozoa were observed [21], ICSI was performed after the provision of informed consent. Fertilized eggs were cultured in continuous single culture medium (Irvine Scientific) with $10 \%$ serum substitute supplement (Irvine Scientific) until day 7 or when fresh embryo transfer was performed. The relationship between the ejaculated motile sperm concentration and fertilization rate during each IVF cycle was analyzed according to the split IVF-ICSI criteria set by Hashimoto et al. [22]. 


\section{Swim-up spermatozoa analysis at 22 hours after insemination}

At 22 hours after insemination, the remaining swim-up spermatozoa were examined at least twice per sample using a Makler Counting Chamber and divided into six groups according to their MSPPI (group A, <10\%; group B, $10 \%$ to <30\%; group C, $30 \%$ to $<50 \%$; group D, $50 \%$ to $<70 \%$; group $E, 70 \%$ to $<90 \%$; and group $F, 90 \%$ to $100 \%)$. MSPPI was calculated as the ratio between the motile spermatozoa (progressive motile and non-progressive motile spermatozoa) [21] and total spermatozoa (motile and immotile spermatozoa) [21] at 22 hours post-insemination. To prevent bias in human analysis of the retrieved samples and to ensure objectivity, the category of motile sperm included even spermatozoa in which only a flagellar beat could be observed, according to the World Health Organization Laboratory Manual for the Examination and Processing of Human Semen [21].

\section{Cryopreservation/thawing/blastocyst transfer}

Blastocysts were cryopreserved and thawed using the Cryotop technique, as previously described [23], with the modification that thawing was performed at room temperature. One frozen-thawed blastocyst was transferred to the uterus of each patient. Luteal support for FBT cycles was performed as previously described [24]. Briefly, patients received $2 \mathrm{mg}$ of chlormadinone acetate (Lutoral, Shionogi \& Co.) three times daily after ovulation, and $0.72 \mathrm{mg}$ transdermal estradiol patches (Estrana Tape, Hisamitsu Pharmaceutical Co., Tokyo, Japan) were applied to the abdomen for 10 weeks after the initiation of menstruation. On the day of blastocyst transfer, if their serum progesterone concentration was $<15 \mathrm{ng} / \mathrm{mL}$, the women were injected with $125 \mathrm{mg}$ of $17 \mathrm{a}$-hydroxyprogesterone caproate once every 5 days. Moreover, patients took a $5 \mathrm{mg}$ prednisolone tablet (Towa Pharmaceutical Co., Osaka, Japan) three times daily for 3 days after ovulation. The dosage of prednisolone was set at $15 \mathrm{mg} /$ day based on a previous study [19]. Stimulation of the endometrium for embryo transfer [25] was performed 2 or 3 days before FBT.

\section{Statistical analysis}

Correlations between the fertilization rate and ejaculated motile sperm concentration and the fertilization rate and MSPPI were evaluated using the Spearman rank-order correlation coefficient (Spearman correlation, $\gamma s$ ). The differences between the mean age of women, the mean number of oocytes recovered, and the number of motile spermatozoa in the original semen and after preparation were evaluated using one-factor analysis of variance. The mean age of the women was evaluated using the Tukey-Kramer post hoc test, and the mean number of oocytes recovered and number of motile spermatozoa were evaluated using the Steel-Dwass method. These data are presented as mean \pm standard deviation. The TFF, fertilization, blastocyst development, chemical pregnancy, clinical pregnancy, and live birth rates were evaluated using the chi-square test and residual analysis. A $p$-value of $<0.05$ was considered to indicate statistical significance.

\section{Results}

This study showed no correlation between the ejaculated motile sperm concentration $\left(0.6-280 \times 10^{6} / \mathrm{mL}\right.$ motile spermatozoa) and fertilization rate in 260 IVF cycles $(\gamma s=-0.079, P=0.357$ ) (Figure 1). Moreover, we analyzed the relationship between the frequency of a $<30 \%$ fertilization rate and sperm concentration or motile sperm percentage in original semen according to the criteria of Hashimoto et al. [22] (an original sperm concentration of $<20 \times 10^{6} / \mathrm{mL}$ or original motile sperm percentage of $<20 \%$ ), which were based on a $<30 \%$ fertilization rate. Our results did not meet these criteria $(<20$ vs. $\geq 20 \times 10^{6} / \mathrm{mL}$ sperm concentration in original semen, $5 / 29$ cycles [17.2\%] vs. 26/231 cycles [11.3\%], $p=0.348$, respectively; and $<20 \%$ vs. $\geq 20 \%$ motile sperm percentage in original semen, $2 / 6$ cycles [33.3\%] vs. 29/254 cycles [11.4\%], $p=0.102$, respectively; data not shown).

Next, we investigated the relationship between the fertilization rate and MSPPI. No significant differences in patient backgrounds, except in the mean number of oocytes recovered (the mean age of the women and the number of motile spermatozoa in the original semen and after preparation were not significantly different), among

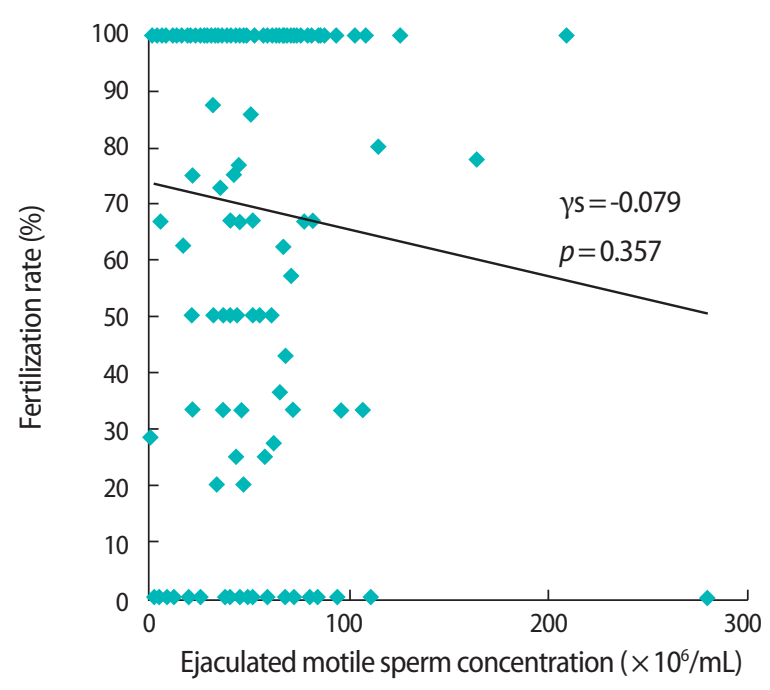

Figure 1. The correlation between ejaculated motile sperm concentration and fertilization rate during each in vitro fertilization cycle. The correlation was evaluated using the Spearman rank-order correlation coefficient ( $n=260$ in vitro fertilization cycles). 
Table 1. Patient backgrounds

\begin{tabular}{|c|c|c|c|c|c|c|}
\hline \multirow[b]{2}{*}{ Variable } & \multicolumn{6}{|c|}{ Motile swim-up spermatozoa percentage at 22 hours post-insemination } \\
\hline & $\begin{array}{l}\text { Group A } \\
(<10 \%)\end{array}$ & $\begin{array}{c}\text { Group B } \\
(10 \% \text { to }<30 \%)\end{array}$ & $\begin{array}{c}\text { Group C } \\
(30 \% \text { to }<50 \%)\end{array}$ & $\begin{array}{c}\text { Group D } \\
\text { (50\% to }<70 \%)\end{array}$ & $\begin{array}{c}\text { Group E } \\
\text { (70\% to }<90 \%)\end{array}$ & $\begin{array}{c}\text { Group } F \\
\text { (90\% to } 100 \%)\end{array}$ \\
\hline No. of IVF cycles & 58 & 28 & 30 & 46 & 51 & 47 \\
\hline Age of women (yr) & $37.3 \pm 5.5$ & $35.2 \pm 5.3$ & $38 \pm 4.4$ & $37.2 \pm 4.7$ & $35.9 \pm 5.2$ & $35.8 \pm 5.4$ \\
\hline No. of oocytes recovered & $4.2 \pm 36.3$ & $4.1 \pm 2.9$ & $5 \pm 4.6$ & $3.5 \pm 3.8^{\mathrm{a}, \mathrm{b})}$ & $5.6 \pm 4.4^{\mathrm{a})}$ & $6.5 \pm 5.7^{b)}$ \\
\hline $\begin{array}{l}\text { No. of motile spermatozoa } \\
\text { in original semen }\left(\times 10^{6} / \mathrm{mL}\right)\end{array}$ & $\begin{array}{r}47 \pm 24.2 \\
(1.9-164)\end{array}$ & $\begin{array}{c}43.3 \pm 23.7 \\
(3.4-97)\end{array}$ & $\begin{array}{c}65.3 \pm 58.1 \\
(2-280)\end{array}$ & $\begin{array}{c}47.7 \pm 18.6 \\
(0.6-104)\end{array}$ & $\begin{array}{c}57.7 \pm 23.2 \\
(14-115)\end{array}$ & $\begin{array}{c}57.1 \pm 25.9 \\
(7.4-117)\end{array}$ \\
\hline $\begin{array}{l}\text { No. of motile spermatozoa } \\
\text { after preparation }\left(\times 10^{6} / \mathrm{mL}\right)\end{array}$ & $21.4 \pm 19.1$ & $15.4 \pm 9.4$ & $21.7 \pm 17.6$ & $15.8 \pm 7.9$ & $20.5 \pm 15.8$ & $17.6 \pm 8.3$ \\
\hline
\end{tabular}

Values are presented as mean \pm standard deviation or mean \pm standard deviation (range). The mean age of the women, the number of recovered oocytes, and the number of motile spermatozoa in the original semen and after preparation were evaluated using one-factor analysis of variance. Consequently, the mean age of the women was evaluated using the Tukey-Kramer post hoc test, and the mean number of oocytes recovered and the number of motile spermatozoa were evaluated using the Steel-Dwass method.

IVF, in vitro fertilization.

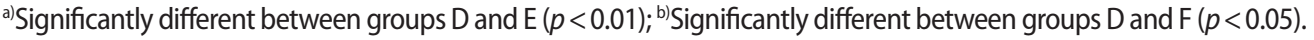

Table 2. The relationship between fertilization rates and motile swim-up spermatozoa percentage at 22 hours post-insemination

\begin{tabular}{|c|c|c|c|c|c|c|c|}
\hline \multirow[b]{2}{*}{ Variable } & \multicolumn{7}{|c|}{ Motile swim-up spermatozoa percentage at 22 hours post-insemination } \\
\hline & $\begin{array}{l}\text { Group A } \\
(<10 \%)\end{array}$ & $\begin{array}{c}\text { Group B } \\
(10 \% \text { to }<30 \%)\end{array}$ & $\begin{array}{c}\text { Group C } \\
\text { (30\% to }<50 \%)\end{array}$ & $\begin{array}{c}\text { Group D } \\
\text { (50\% to }<70 \%)\end{array}$ & $\begin{array}{c}\text { Group E } \\
\text { (70\% to }<90 \%)\end{array}$ & $\begin{array}{c}\text { Group F } \\
\text { (90\% to 100\%) }\end{array}$ & $p$-value \\
\hline No. of IVF cycles & 58 & 28 & 30 & 46 & 51 & 47 & - \\
\hline Total fertilization failures & $15(25.9)^{*}$ & $6(21.4)$ & $3(10)$ & $2(4.3)$ & $2(3.9)$ & $1(2.1)^{*}$ & 0.035 \\
\hline Oocyte recovered & 208 & 90 & 109 & 133 & 233 & 262 & - \\
\hline 2PN2PB oocyte & $107(51.4)^{* *}$ & $47(52.2)^{*}$ & $73(67)$ & $85(63.9)$ & $159(68.2)$ & $185(70.6)^{*}$ & $<0.001$ \\
\hline 1PN3PB oocyte & $7(3.4)$ & $3(3.3)$ & $2(1.8)$ & $6(4.5)$ & $5(2.1)$ & $6(2.3)$ & 0.737 \\
\hline Polyspermy oocyte & $0^{*}$ & 0 & $3(2.8)$ & $2(1.5)$ & $9(3.9)^{*}$ & $6(2.3)$ & 0.049 \\
\hline OPN2PB oocyte & $16(7.7)$ & $3(3.3)$ & $12(11)$ & $10(7.5)$ & $21(9)$ & $23(8.8)$ & 0.488 \\
\hline Unfertilized egg & $78(37.5)^{* * *}$ & $37(41.1)^{* * *}$ & $19(17.4)$ & $30(22.6)$ & $39(16.7)^{* * *}$ & $42(16)^{* * *}$ & $<0.001$ \\
\hline
\end{tabular}

Values are presented as number (\%). Rates of total fertilization failure, 2PN2PB, 1PN3PB, polyspermy, OPN2PB oocytes, and unfertilized eggs were evaluated using the chi-square test and residual analysis. A probability level of $p<0.05$ was considered to indicate statistical significance.

IVF, in vitro fertilization; $\mathrm{PN}$, pronucleus; $\mathrm{PB}$, polar body.

${ }^{*} p<0.05 ;{ }^{* *} p<0.01$.

Table 3. Blastocyst development and embryo transfer outcome

\begin{tabular}{|c|c|c|c|c|c|c|c|}
\hline \multirow[b]{2}{*}{ Variable } & \multicolumn{7}{|c|}{ Motile swim-up spermatozoa percentage at 22 hours post-insemination } \\
\hline & $\begin{array}{l}\text { Group A } \\
(<10 \%)\end{array}$ & $\begin{array}{c}\text { Group B } \\
(10 \% \text { to }<30 \%)\end{array}$ & $\begin{array}{c}\text { Group C } \\
\text { (30\% to }<50 \%)\end{array}$ & $\begin{array}{c}\text { Group D } \\
\text { (50\% to }<70 \%)\end{array}$ & $\begin{array}{c}\text { Group E } \\
\text { (70\% to }<90 \%)\end{array}$ & $\begin{array}{c}\text { Group F } \\
\text { (90\% to } 100 \%)\end{array}$ & $p$-value \\
\hline No. of cultured oocytes & 78 & 32 & 59 & 57 & 130 & 160 & - \\
\hline Blastocyst & $45(57.7)$ & $19(59.4)$ & $37(62.7)$ & $32(56.1)$ & $60(46.2)$ & $94(58.8)$ & 0.230 \\
\hline No. of fresh embryo transfers & 21 & 12 & 11 & 26 & 22 & 23 & - \\
\hline Chemical pregnancy & $7(33.3)$ & 0 & $3(27.3)$ & $5(19.2)$ & $4(18.2)$ & $4(17.4)$ & 0.321 \\
\hline Clinical pregnancy & $3(14.3)$ & 0 & $2(18.2)$ & $2(7.7)$ & $3(13.6)$ & $1(4.3)$ & 0.549 \\
\hline Live birth & 0 & 0 & $2(18.2)$ & 0 & $1(4.5)$ & $1(4.3)$ & 0.096 \\
\hline No. of frozen-thawed blastocyst transfers & 31 & 10 & 23 & 19 & 43 & 49 & - \\
\hline Chemical pregnancy & $14(45.2)$ & $6(60)$ & $8(34.8)$ & $8(42.1)$ & $19(44.2)$ & $22(44.9)$ & 0.863 \\
\hline Clinical pregnancy & $10(32.3)$ & $5(50)$ & $7(30.4)$ & $8(42.1)$ & $14(32.6)$ & $12(24.5)$ & 0.836 \\
\hline Live birth & $7(22.6)$ & $5(50)$ & $4(17.4)$ & $7(36.8)$ & $8(18.6)$ & $12(24.5)$ & 0.228 \\
\hline
\end{tabular}

Values are presented as number (\%). Rates of blastocyst development, chemical pregnancy, clinical pregnancy, and live births were evaluated using the chisquare test and residual analysis. A probability level of $p<0.05$ was considered to indicate statistical significance. 


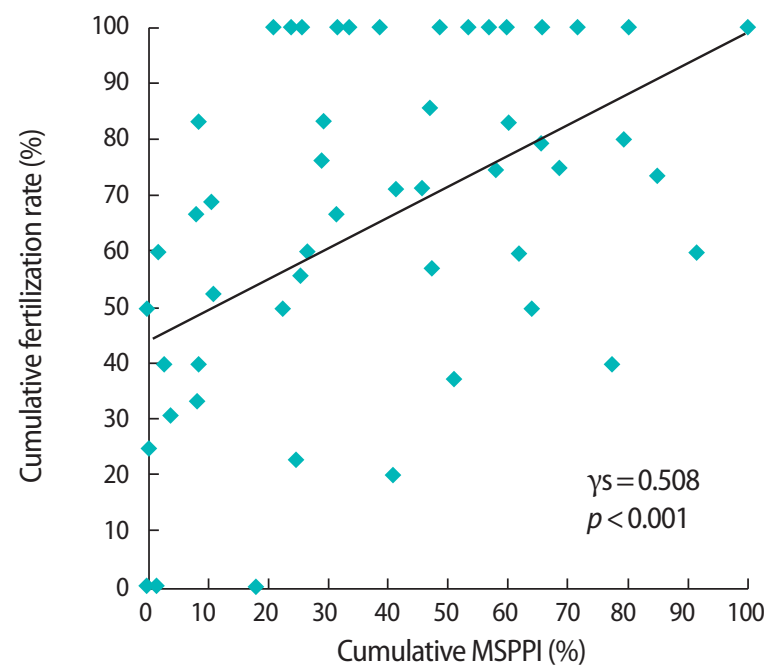

Figure 2. The correlation between cumulative motile swim-up spermatozoa percentage at 22 hours post-insemination and cumulative fertilization rate throughout all cycles in patients who received at least two in vitro fertilization cycles. The correlation was evaluated using the Spearman rank-order correlation coefficient $(n=52$ patients, 148 in vitro fertilization cycles).

the groups (Table 1). The mean number of oocytes recovered was significantly lower in group $D$ than in groups $E$ and $F$ (Table 1). The incidence of TFF was significantly higher in group $A$ than in the other groups ( $p=0.035$ ). In groups $A$ and $B$, the occurrence of two pronuclei and two polar bodies (2PN2PB) was significantly lower than in groups $C$ to $F(p=0.00007)$. Moreover, in groups $A$ and $B$, the occurrence of unfertilized eggs was significantly higher than in the other groups $(p<0.0001)$. Conversely, the incidence of TFF in group F was significantly lower than in the other groups $(p=0.035)$ and the occurrence of 2PN2PB in group F was significantly higher than in the other groups $(p=0.00007)$. Also, the occurrence of unfertilized eggs in groups $\mathrm{E}$ and $\mathrm{F}$ was significantly lower than in the other groups $(p<0.001)$ (Table 2). Regarding embryo development, no significant differences were evident in the rates of blastocyst development, chemical pregnancy, clinical pregnancy, and live birth among the groups (Table 3).

We found a correlation between the cumulative MSPPI and cumulative fertilization rate in patients who underwent at least two cycles $(\gamma s=0.508, p<0.001)$ (Figure 2).

\section{Discussion}

In this study, we showed that cumulative MSPPI is correlated with the cumulative fertilization rate. These results suggest that a low MSPPI value is a potential indicator for split IVF-ICSI and ICSI.

We began our study by investigating the correlation between the ejaculated motile sperm concentration and the fertilization rate during each IVF cycle. We showed that the fertilization rate was not correlated with the ejaculated motile sperm concentration. We evaluated our data using the criteria of Hashimoto et al. [22], and our findings were not consistent with theirs. This suggests that basing the decision to use IVF, ICSI, or split IVF-ICSI on the motile sperm concentration may lead to unnecessary ICSI.

In terms of predicting the fertility of cultured swim-up spermatozoa, our method was similar to SST; however, there were some differences in our calculation method. In particular, SST calculates the ratio between the final concentration of progressive spermatozoa after 24 hours and the initial concentration of progressive spermatozoa [17]. Our method, however, calculated the ratio between the motile spermatozoa and total spermatozoa at 22 hours post-insemination.

Our results showed that, regardless of the ejaculated motile sperm concentration, the incidence of TFF significantly increases when MSPPI is $<10 \%$ and that the fertilization rate significantly decreases when MSPPI is $<30 \%$. Tournaye et al. [2] reported that the incidence of TFF is higher when insemination is performed with 5,000 sperm/ oocyte than when performed with 20,000 sperm/oocyte $(25.7 \%$ vs. $5.3 \%)$. In this study, despite using an insemination concentration of $>20,000$ spermatozoa/oocyte, TFF occurred in $25.9 \%$ of patients when MSPPI was $<10 \%$. Accordingly, the main cause of TFF in this study is unlikely to be the number of motile spermatozoa/oocyte. Regarding embryo development, no significant differences were evident in the rates of blastocyst development, chemical pregnancy, clinical pregnancy, and live birth among the groups. These results suggest that MSPPI relates to the fertilization rate alone.

After sperm preparation techniques, such as swim-up procedure and density gradient centrifugation, intercellular reactive oxygen species (ROS) and hydrogen peroxide concentrations and DNA fragmentation in human spermatozoa increase [26,27]. Tremellen [28] reported that ROS damage the sperm membrane, thus decreasing the motility of sperm and their ability to fuse with the oocyte. Also, ROS can alter sperm DNA, resulting in the passage of defective paternal DNA to the conceptus.

Moreover, ROS levels are higher in the semen of patients with astheno teratozoospermia and leukocytospermia than in those of normozoospermic individuals [29]. In a meta-analysis, Agarwal et al. [30] reported that fertilization rates after IVF correlate with seminal ROS levels. Our data showed a correlation between MSPPI and fertilization rate. We speculate that the resistance of spermatozoa to ROS may be an important factor in assisted reproductive technology.

There are some limitations in the present study. The first is that the analysis was based on cumulative data that were obtained from the relationship between fertilization and MSPPI in each patient as the main results. The data are obtained from two IVF cycles in cases of 
complete IVF failure, because we were investigating TFF patients who repeatedly failed to fertilize by IVF. An ICSI approach is a top priority method in the third cycle. A second limitation was that sperm analysis performed by embryologists may be less objective than computer-aided sperm analysis [31,32]. However, to ensure objectivity, the category of motile sperm included even spermatozoa in which only a flagellar beat can be observed. Thus, we prevented bias in human analysis of sperm.

In conclusion, MSPPI may be a viable indicator for split IVF-ICSI and ICSI. By employing the MSPPI test during semen analysis and/or artificial insemination, unnecessary split IVF-ICSI and ICSI may be avoided.

\section{Conflict of interest}

No potential conflict of interest relevant to this article was reported.

\section{References}

1. Roest J, Van Heusden AM, Zeilmaker GH, Verhoeff A. Treatment policy after poor fertilization in the first IVF cycle. J Assist Reprod Genet 1998;15:18-21.

2. Tournaye H, Verheyen G, Albano C, Camus M, Van Landuyt L, Devroey $\mathrm{P}$, et al. Intracytoplasmic sperm injection versus in vitro fertilization: a randomized controlled trial and a meta-analysis of the literature. Fertil Steril 2002;78:1030-7.

3. Bungum L, Bungum M, Humaidan P, Andersen CY. A strategy for treatment of couples with unexplained infertility who failed to conceive after intrauterine insemination. Reprod Biomed Online 2004;8:584-9.

4. Liu DY, Baker HW. Defective sperm-zona pellucida interaction: a major cause of failure of fertilization in clinical in-vitro fertilization. Hum Reprod 2000;15:702-8.

5. Kuczynski W, Dhont M, Grygoruk C, Pietrewicz P, Redzko S, Szamatowicz M. Rescue ICSI of unfertilized oocytes after IVF. Hum Reprod 2002;17:2423-7.

6. Tesarik J. Associate editor's comment on 'Rescue ICSI of oocytes that failed to extrude the second polar body $6 \mathrm{~h}$ post-insemination in conventional IVF' by Chen and Kattera: rescue ICSI revisited. Hum Reprod 2003;18:2122-3.

7. Chen C, Kattera S. Rescue ICSI of oocytes that failed to extrude the second polar body $6 \mathrm{~h}$ post-insemination in conventional IVF. Hum Reprod 2003;18:2118-21.

8. Nagy ZP, Rienzi LF, Ubaldi FM, Greco E, Massey JB, Kort HI. Effect of reduced oocyte aging on the outcome of rescue intracytoplasmic sperm injection. Fertil Steril 2006;85:901-6.

9. Hershlag A, Paine T, Kvapil G, Feng H, Napolitano B. In vitro fertilization-intracytoplasmic sperm injection split: an insemination method to prevent fertilization failure. Fertil Steril 2002;77:22932.

10. European Society of Human Reproduction and Embryology. Good clinical treatment in assisted reproduction: an ESHRE position paper. Belgium: ESHRE; 2008.

11. Practice Committees of the American Society for Reproductive Medicine and Society for Assisted Reproductive Technology. Intracytoplasmic sperm injection (ICSI) for non-male factor infertility: a committee opinion. Fertil Steril 2012;98:1395-9.

12. Japan Society of Obstetrics and Gynecology. Kenbijusei ni kansuru kenkai. Acta Obstet Gynaecol Jpn 2014;8:1877.

13. Shalom-paz E, Alshalati J, Shehata F, Jimenez L, Son WY, Holzer H, et al. Clinical and economic analysis of rescue intracytoplasmic sperm injection cycles. Gynecol Endocrinol 2011;27:993-6.

14. Liu W, Liu J, Zhang X, Han W, Xiong S, Huang G. Short co-incubation of gametes combined with early rescue ICSI: an optimal strategy for complete fertilization failure after IVF. Hum Fertil (Camb) 2014;17:50-5.

15. Vitek WS, Galarraga O, Klatsky PC, Robins JC, Carson SA, Blazar AS. Management of the first in vitro fertilization cycle for unexplained infertility: a cost-effectiveness analysis of split in vitro fertilization-intracytoplasmic sperm injection. Fertil Steril 2013; 100:1381-8.

16. Coccia ME, Becattini C, Criscuoli L, Fuzzi B, Scarselli G. A sperm survival test and in-vitro fertilization outcome in the presence of male factor infertility. Hum Reprod 1997;12:1969-73.

17. Sukcharoen N, Keith J. Evaluation of the percentage of sperm motility at $24 \mathrm{~h}$ and sperm survival ratio for prediction of in vitro fertilization. Andrologia 1996;28:203-10.

18. Eskandar M. Is 24-h sperm motility a useful IVF measure when male infertility is not apparent? Acta Obstet Gynecol Scand 2002;81:328-30.

19. Inoue T, Ono Y, Yonezawa Y, Kishi J, Emi N. Improvement of live birth rate following frozen-thawed blastocyst transfer by combination of prednisolone administration and stimulation of endometrium embryo transfer. Open J Obstet Gynecol 2014;4:74550.

20. Inoue T, Ono Y, Yonezawa Y, Oi M, Kobayashi N, Kishi J, et al. Oocyte quality improvement using a herbal medicine comprising 7 crude drugs. Open J Obstet Gynecol 2013;3:195-202.

21. World Health Organization. WHO laboratory manual for the examination and processing of human semen. 5th ed. Zeneva: World Health Organization; 2010.

22. Hashimoto H, Goto S, Tsubouchi M, Izumi Y, Yoshimura Y, Kasahara Y, et al. Indication for split ICSI in our clinic. J Mamm Ova Res 2004;21:209-13.

23. Kuwayama M. Highly efficient vitrification for cryopreservation 
of human oocytes and embryos: the Cryotop method. Theriogenology 2007;67:73-80.

24. Inoue T, Ono Y, Emi N. Preservation of spermatozoa by refrigeration. In: Frias TA, Cano MJ, editors. Pregnancy: risk factors, management and recovery. New York: Nova Science Publishers; 2012. p.121-46.

25. Goto S, Kadowaki T, Hashimoto H, Kokeguchi S, Shiotani M. Stimulation of endometrium embryo transfer can improve implantation and pregnancy rates for patients undergoing assisted reproductive technology for the first time with a high-grade blastocyst. Fertil Steril 2009;92:1264-8.

26. Mortimer D. Sperm preparation methods. J Androl 2000;21:35766.

27. Ghaleno LR, Valojerdi MR, Janzamin E, Chehrazi M, Sharbatoghli $M$, Yazdi RS. Evaluation of conventional semen parameters, intracellular reactive oxygen species, DNA fragmentation and dysfunction of mitochondrial membrane potential after semen preparation techniques: a flow cytometric study. Arch Gynecol Obstet 2014;289:173-80.

28. Tremellen K. Oxidative stress and male infertility: a clinical perspective. Hum Reprod Update 2008;14:243-58.

29. Li Z, Zhou Y, Liu R, Lin H, Liu W, Xiao W, et al. Effects of semen processing on the generation of reactive oxygen species and mitochondrial membrane potential of human spermatozoa. Andrologia 2012;44:157-63.

30. Agarwal A, Allamaneni SS, Nallella KP, George AT, Mascha E. Correlation of reactive oxygen species levels with the fertilization rate after in vitro fertilization: a qualified meta-analysis. Fertil Steril 2005;84:228-31.

31. Lu JC, Huang YF, Lu NQ. Computer-aided sperm analysis: past, present and future. Andrologia 2014;46:329-38.

32. Mortimer ST, van der Horst G, Mortimer D. The future of computer-aided sperm analysis. Asian J Androl 2015;17:545-53. 